SHORT REPORT

\title{
Phenotypic variability in a Chinese family with rimmed vacuolar distal myopathy
}

\author{
L-S Ro, G-J Lee-Chen, Y-R Wu, M Lee, P-Y Hsu, C-M Chen
}

J Neurol Neurosurg Psychiatry 2005;76:752-755. doi: 10.1136/jnnp.2004.048876

Background: UDP-N-acetylglucosamine 2-epimerase/Nacetylmannosamine kinase (GNE) gene mutations have been found in patients with distal myopathy with rimmed vacuoles (DMRV). It is not clear how the same GNE gene mutations can result in different phenotypes in the same family with DMRV.

Methods: The clinical, neurophysiological, histopathological, and genetic characteristics of two patients with DMRV from a Chinese family from Taiwan were investigated.

Results: Two novel compound heterozygous mutations in different domains of the protein, lle241Ser in the epimerase and Trp513stop in the kinase domain, were detected in both patients. However, the two patients demonstrated different patterns of disease progression: one had slow disease progression with a typical feature of DMRV (that is, weakness beginning in the distal leg muscles, typically anterior tibialis, with the quadriceps remaining relatively unaffected), and the other had rapid disease progression with an atypical presentation of DMRV.

Conclusions: The results of the present study indicate that GNE gene mutations and probably modifier gene(s) or additional factors may result in different phenotypes of DMRV.

$\mathrm{D}$ istal myopathy with rimmed vacuoles (DMRV) is characterised by ${ }^{1-3}$ :

- autosomal recessive inheritance but may also occur sporadically

- onset of symptoms in early adulthood, generally between 20 and 30 years of age

- weakness beginning in the distal leg muscles, typically in the anterior tibialis muscle, with the quadriceps muscle remaining relatively unaffected

- mainly myogenic changes, with certain neurogenic features on electromyography (EMG)

- normal or mildly elevated serum creatine kinase (CK)

- muscles biopsies displaying rimmed vacuoles without obvious dystrophic features.

Another hereditary inclusion body myopathy (HIBM) has clinical and histopathological characteristics similar to DMRV, and it has been reported mainly from the Middle East. Recently, UDP- $N$-acetylglucosamine 2-epimerase/ $N$ acetylmannosamine kinase (GNE) gene mutations have been identified in patients with autosomal recessive $\mathrm{HIBM}^{5}$ and DMRV. ${ }^{6}$ Therefore, HIBM and DMRV may be the same disease entity rather than two allelic disorders. ${ }^{6}$ To clarify whether DMRV in Chinese is also caused by GNE gene mutations, we investigated clinical and genetic characteristics of two Chinese patients with DMRV.

\section{PATIENTS AND METHODS}

We examined two patients with DMRV from a Chinese family living in Chung-Hwa, southern Taiwan. Informed consent was obtained from each member of the family, and the institutional review board of Chang Gung Memorial Hospital approved the study.

\section{Case II-2}

A 50-year-old man developed an insidious steppage gait at 30 years of age. Within four years, he was unable to stand on his heels and climb stairs. By the age of 36, the patient had difficulty rising up from a sitting position. Except his younger sister (case II-3), no other family member had a similar condition.

Neurological examination revealed muscle wasting in both the upper and lower extremities, particularly the distal parts. Severe weakness was observed in the tibialis anterior, extensor digitorum and hallucis muscles, along with moderate weakness in the gluteus minimus and adductor thigh muscles, and mild weakness in the upper extremities and the neck flexor muscles. Ankle and triceps jerks were absent, but the knee, biceps, and brachioradialis jerks were preserved. The rest of the neurological examination was unremarkable.

EMG showed myopathic patterns with some neuropathic changes; serum CK was twice the normal level. On light microscopy, the muscle biopsy from the quadriceps femoris showed variations in fibre size with scattered, angular, and hypertrophic fibres and mild increase of endomysial fibrosis when stained with haematoxylin and eosin. Notably, purplered rimmed vacuoles were observed with Gomori trichrome staining. Electron microscopy revealed several autophagic vacuoles containing various lamellar structures and some cytoplasmic filamentous inclusions. Magnetic resonance imaging (MRI) demonstrated fibrotic, fatty, and atrophic changes in the anterior compartment muscles of the lower legs and to a lesser degree in the posterior compartment muscles. The thigh hamstrings, adductor, and gracilis muscles were severely involved, but the quadriceps femoris muscles were spared (table 1 and fig 1).

\section{Case II-3}

A 41-year-old woman, the younger sister of case II-2, began to experience difficulty standing up at 26 years of age. She was unable to climb stairs one year later and was wheelchair bound by 31 years of age.

On examination, the patient displayed marked weakness and atrophy in the distal parts of her legs. The proximal muscles were also atrophic, particularly in the lower extremities. The neck, shoulder girdle, arm, and hand muscles also showed mild to moderate weakness. All tendon reflexes were absent. EMG displayed myopathic changes in

Abbreviations: DMRV, distal myopathy with rimmed vacuoles; HIBM, hereditary inclusion body myopathy; GNE, UDP-N-acetylglucosamine 2epimerase/ $\mathrm{N}$-acetylmannosamine kinase 
Table 1 Clinical and laboratory findings of Nonaka distal myopathy in two Chinese patients

\begin{tabular}{|c|c|c|}
\hline & Case II-2 & Case II-3 \\
\hline Sex & Male & Female \\
\hline Age, years & 50 & 41 \\
\hline Age of onset, years & 30 & 26 \\
\hline Inheritance & Autosomal recessive & Autosomal recessive \\
\hline Initial weakness & Foot drop & Foot drop \\
\hline \multicolumn{3}{|l|}{ Distribution of weakness and severity } \\
\hline Severe & Anterior distal legs & Anterior and posterior distal legs \\
\hline Moderate & Posterior distal legs and posterior thigh & Anterior and posterior thigh, upper limbs \\
\hline Mild & Upper limbs & Neck flexor muscles \\
\hline \multicolumn{3}{|l|}{ Medical Research Council (MRC) scales } \\
\hline Quadriceps & 5 & 2 \\
\hline Hamstrings & 4 & 1 \\
\hline Tibialis anterior & 1 & 0 \\
\hline Gastrocnemius & 4 & 1 \\
\hline Quadriceps sparing & Yes & No \\
\hline Creatine kinase (reference range 50-130 IU/I) & 322 & 177 \\
\hline \multirow{2}{*}{\multicolumn{3}{|c|}{ Magnetic resonance imaging fatty infiltration* }} \\
\hline & & \\
\hline Quadriceps & - & $++\sim++$ \\
\hline Hamstrings & $+\sim++$ & $+++\sim++++$ \\
\hline Tibialis anterior & $+\sim++$ & +++ \\
\hline Gastrocnemius & $-\sim+$ & $+++\sim++++$ \\
\hline Muscle biopsy & $\begin{array}{l}\text { Mild variation in fibre size, many rimmed } \\
\text { vacuoles }\end{array}$ & Mild variation in fibre size, many rimmed vacuoles \\
\hline Current status & Walks with assistance & Wheelchair bound \\
\hline
\end{tabular}

all tested muscles; serum CK was slightly higher than the normal level. A muscle biopsy revealed characteristic findings of myopathy, with numerous rimmed vacuoles on light microscopy. An MRI showed significant degree of fatty, fibrotic, and atrophic changes in the distal and proximal parts of the lower extremities. The quadriceps muscles showed a moderate degree of involvement (see table 1 and fig 1).

\section{DNA analysis}

DNA was extracted from peripheral blood leucocytes using a DNA extraction kit (Stratagene; La Jolla, CA). Eleven coding exons (exons 2-12) and flanking sequences of the GNE gene were amplified by polymerase chain reaction (PCR) using
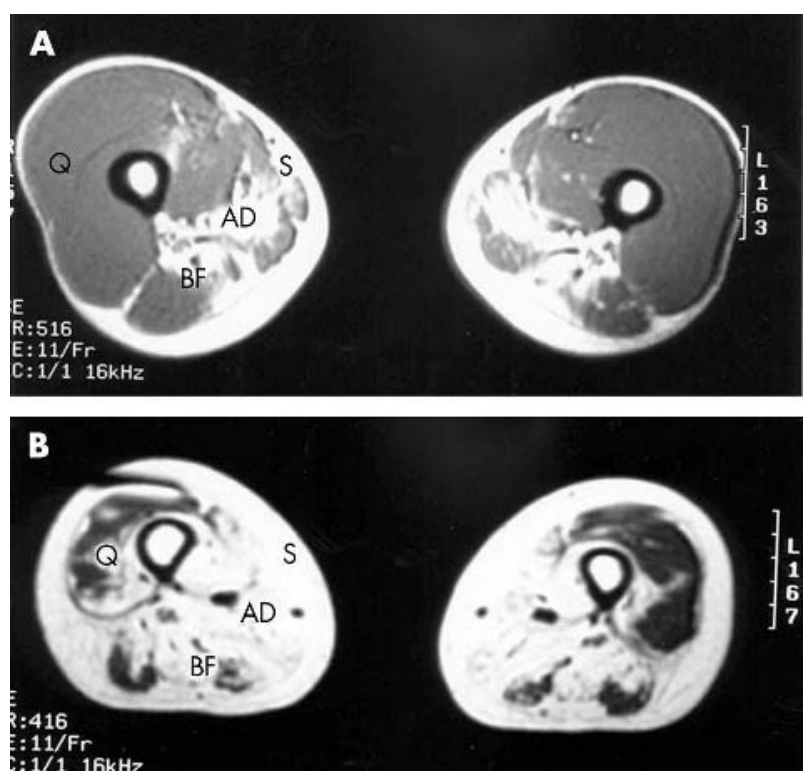

Figure 1 Tl-weighted magnetic resonance imaging (MRI) of the upper legs shows moderate atrophy and increased signal intensities in the adductor longus and adductor magnus (D), biceps femoris (BF) and sartorius $(S)$ muscles. The quadriceps $(Q)$ muscle is well preserved in case II-2 (A), but not in case II-3 (B). primers and conditions as previously described. ${ }^{5}$ The PCR products were subjected to sequencing using an automated DNA sequencer (MegaBACE Analyzer; Molecular Dynamics, Division of Amersham Pharmacia Biotech, Buckingham, England).

\section{RESULTS}

\section{Haplotype analysis}

The family was genotyped using polymorphic markers linked to the GNE gene revealing the chromosome segregating with the disease (fig 2). Haplotype analysis showed that the two affected siblings (II-2 and II-3) had inherited the same chromosomes from their parents, indicating that the disease phenotype was linked to markers flanking the GNE gene (see fig 2).

\section{Detection of the GNE gene mutation}

By screening the 11 exons of the GNE gene, we identified two novel mutations in this family. The Ile24lSer mutation is a $\mathrm{T}$ to $G$ transversion in exon 4, substituting isoleucine for serine at codon 241. Meanwhile, the Trp513stop mutation is a $G$ to A transition in exon 9, converting tryptophan to termination at codon 513 (fig 3). The Ile241Ser mutation was confirmed by $A l u$ I restriction analysis. The amplified exon 4 fragments were digested with $A l u$ I and fractionated on a $2.0 \%$ agarose gel. The mutation created a new AluI restriction site on the PCR product so that, on digestion, 198 and 137 bp fragments appeared instead of wild type $335 \mathrm{bp}$ fragments (see fig 2). Both patients, II-2 and II-3, inherited the Ile241Ser mutation from their mother (I-2) and transmitted it to their offspring (III-1 and III-2). The Trp513stop mutation was inherited paternally (I-1), and one offspring (III-3) also carried the mutation. The Trp513stop mutation predicts a lack of 210 amino acids at the carboxyl terminus of the protein. The novel point mutation Ile241Ser was not observed in 50 healthy Taiwanese subjects.

\section{DISCUSSION}

In some patients with DMRV the disease may progress rapidly, ${ }^{1}$ but in others it may progress slowly. ${ }^{7}$ The two patients reported here showed variations in the clinical course of the disease. The diffuse involvement of the leg 

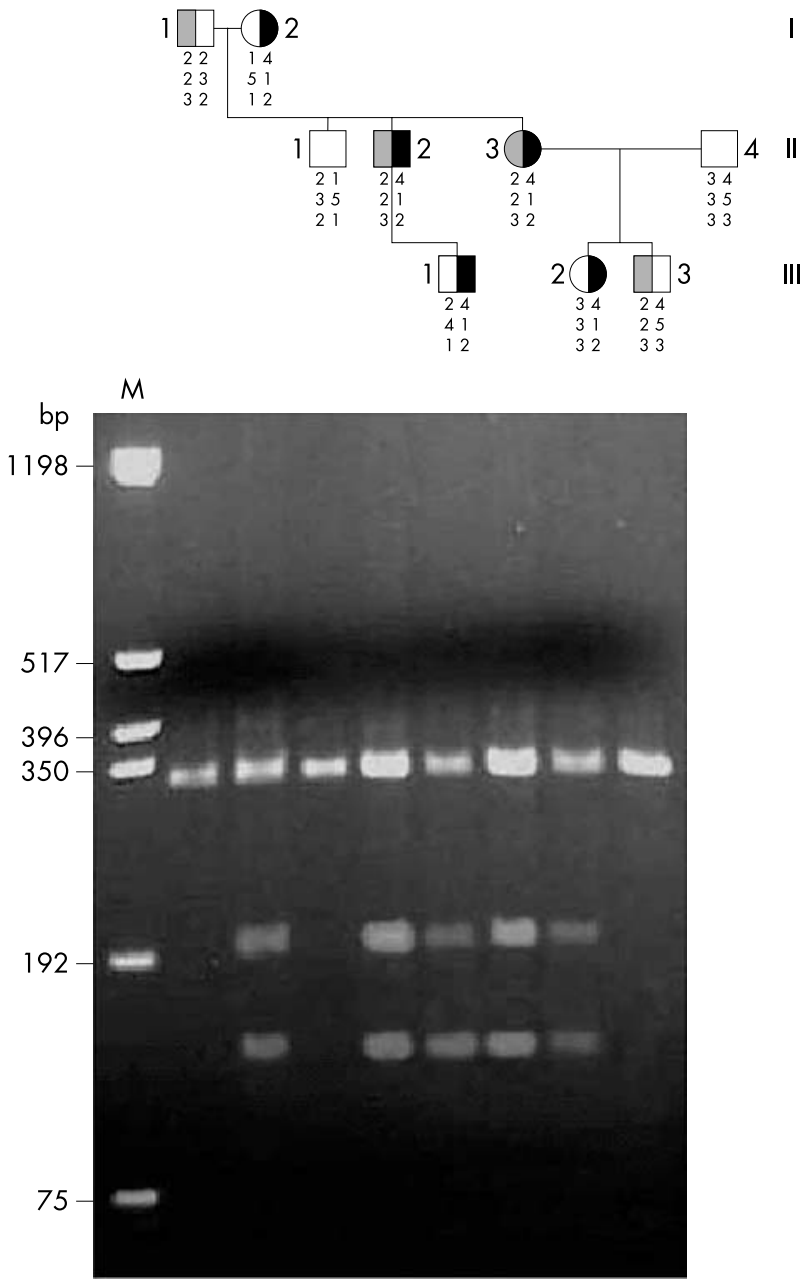

Figure 2 Haplotypes for microsatellite markers D9S1788, D9S1804, and D9S1791 (from top to bottom) are displayed for each member of the Chinese family. Lower panel: DNA polymerase chain reaction (PCR) and restriction enzyme analyses of the lle241Ser allele. Lane $M$ is the size marker. muscles observed clinically in case II-3 was attributed to rapid disease progression. Also, MRI demonstrated severe involvement of both the gastrocnemius and quadriceps femoris muscles in this patient. In contrast, in case II-2, MRI revealed a peculiar distribution of muscle involvement. The quadriceps femoris muscles were well preserved, but the tibialis anterior, the hamstrings, and the adductors were severely affected. Our findings are consistent with a previous study, which showed that the clinical course of DMRV can differ in patients from the same family ${ }^{8}$; however, molecular analysis was not done in that study.

$G N E$, a rate limiting enzyme that catalyses the initial two steps in the biosynthesis of sialic acid, has two functional domains that work independently: the epimerase domain in the amino $(\mathrm{N})$-terminus, and the kinase domain in the carboxy (C)-terminus. ${ }^{9}$ The enzyme catalyses the conversion of UDP- $N$-acetylglucosamine to $N$-acetylmannosamine (ManNAc) and of ManNAc to ManNAc 6-phosphate. It has been shown that sialylation is reduced but not completely absent in muscle and in cultured cells from patients, because all of the mutant proteins with missense mutations partially retained both enzymatic activities. ${ }^{10}$ GNE is expressed in large amounts in liver; therefore, the reduction in enzymatic activities caused by missense mutations may not significantly affect the synthesis of sialic acid in the liver in patients with DMRV, and sialic acid concentrations are comparable with normal blood levels. ${ }^{10}$ In contrast, sialic acid content is reduced in DMRV skeletal muscles. ${ }^{10}$ The reduced enzymatic activities with weak expression of GNE protein are probably responsible for the more serious reduction in sialic acid synthesis in the muscles compared with that in the plasma. Thus, the selective involvement may be a result of muscle uptake of sialic acid, which can compensate for the defective sialic acid synthesis in most muscle fibres.

V572L is the commonest mutation in Japanese patients with $\mathrm{DMRV}^{6}$ and $\mathrm{M712 \textrm {T }}$ is the commonest mutation in Jewish patients with HIBM. ${ }^{6}$ Although several other mutations in the GNE gene have been identified recently, ${ }^{11}$ Ile241Ser and Trp513stop have not been reported previously. Our finding of two novel mutant alleles in a Chinese family with DMRV expands on the genetic heterogeneity of this disease and allows for a broader phenotypic-genotypic

\section{Exon 4 lle241Ser}

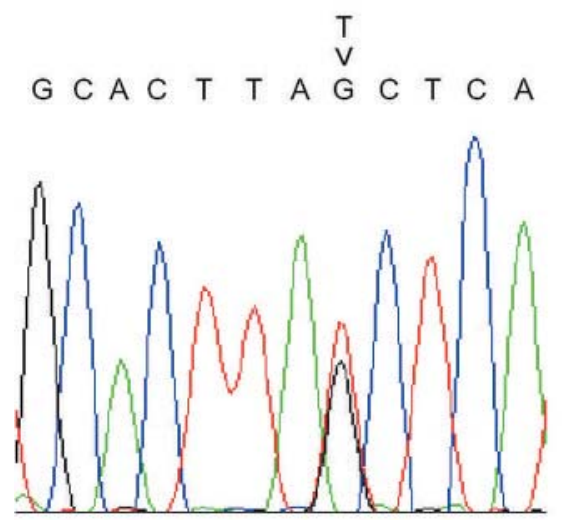

\section{Exon 9 Trp513stop}
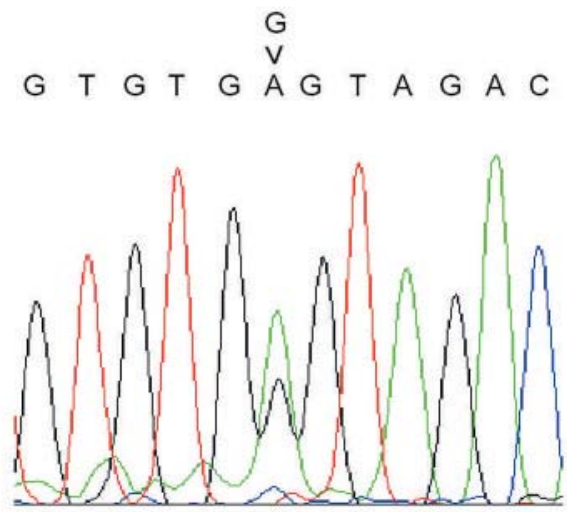

Figure 3 Sequence analysis of polymerase chain reaction products amplified from genomic DNA of an affected individual with distal myopathy with ringed vacuoles (case II-2). The T-to-G transversion at nucleotide 773 in exon 4 results in a conservative amino acid change (lle241Ser) and the G-to-A transition at nucleotide 1590 in exon 9 results in a nonsense mutation (Trp513stop). 
assessment. Tomimitsu et $a l^{6}$ suggested that typical clinical features of DMRV (V572L) and HIBM (M712T) result from homozygous mutations in the kinase domain, whereas involvement of the quadriceps muscles results from compound heterozygous mutations in both the epimerase and kinase domains. However, our patients (from the same family) displayed the same compound heterozygous mutations in different domains of the protein, Ile241Ser in the epimerase domain and Trp513stop in the kinase domain, but demonstrated different phenotypes. The two catalytic domains of the GNE molecule do not always work separately or independently. ${ }^{10}$ For example, although the A524V mutation is within the predicted kinase domain, it strongly inhibited epimerase activity. The present findings suggest that the interaction between the epimerase and kinase activities of the GNE molecule is not as simple as we think. The results also indicate that modifier gene(s) or additional factors might be responsible for the phenotype differences, although no such modifier genes have been identified. Furthermore, an enzyme distinct from GNE, $N$-acetylglucosamine kinase, with $\mathrm{N}$-acetylmannosamine kinase activity, which can compensate the kinase activity of GNE, has been identified recently. ${ }^{12}$ Additionally, it has been reported that, within the same family with HIBM, the quadriceps muscles are not spared in some patients. ${ }^{13}$ In summary, these findings may support our hypothesis. Further clinical, biochemical, and molecular genetic analyses are required to clarify this hypothesis.

\author{
Authors' affiliations \\ L-S Ro, Y-R Wu, M Lee, C-M Chen, Department of Neurology, Chang \\ Gung Memorial Hospital, Taipei, Taiwan \\ G-J Lee-Chen, P-Y Hsu, Department of Life Science, National Taiwan \\ Normal University, Taipei, Taiwan \\ Competing interests: none declared
}

Correspondence to: Dr C-M Chen, Department of Neurology, Chang Gung Memorial Hospital, 199 Tun Hwa North Road, Taipei, Taiwan 10591; cgrols@adm.cgmh.org.tw

Received 30 June 2004

Revised version received 25 August 2004

Accepted 7 September 2004

\section{REFERENCES}

1 Nonaka I, Sunohara N, Ishiura S, et al. Familial distal myopathy with rimmed vacuole and lamellar (myeloid) body formation. J Neurol Sci 1981;51:141-55

2 Mizusawa $\mathbf{H}$, Kurisaki $\mathrm{H}$, Takatsu $\mathrm{M}$, et al. Rimmed vacuolar distal myopathy: a clinical, electrophysiological, histopathology and computed tomographic study of seven cases. J Neurol 1987;234:129-36.

3 Nonaka I, Sunohara N, Satoyoshi E, et al. Autosomal recessive distal muscular dystrophy: a comparative study with distal myopathy with rimmed vacuole formation. Ann Neurol 1985;17:51-9.

4 Mitrani-Rosenbaum S, Argov Z, Blumenfeld A, et al. Hereditary inclusion body myopathy maps to chromosome 9pl-ql. Hum Mol Genet 1996:5:159-63.

5 Eisenberg I, Avidan N, Potikha T, et al. The UDP-N-acetylgluscosamine 2epimerase/ $\mathrm{N}$-acetylmannosamine kinase gene is mutated in recessive hereditary inclusion body myopathy. Nat Genet 2001;29:83-7.

6 Tomimitsu H, Ishikawa K, Shimizu J, et al. Distal myopathy with rimmed vacuoles: Novel mutations in the GNE gene. Neurology 2002:59:451-4.

7 Mizusawa $\mathrm{H}$, Kurisaki $\mathrm{H}$, Takatsu $\mathrm{M}$, et al. Rimmed vacuolar distal myopathy: an ultrastructural study. J Neurol 1987;234:137-45.

8 Markesbery WR, Griggs RC, Herr B. Distal myopathy: electron microscopic and histochemical studies. Neurology 1977;27:727-35.

9 Stäche R, Hinderlich S, Weise C, et al. A bifunctional enzyme catalyzes the first two steps in $N$-acetylneuraminic acid biosynthesis of rat liver. J Biol Chem 1997;272:24319-24.

10 Noguchi S, Keira Y, Murayama K, et al. Reduction of UDP-GlcNAc 2 epimerase/ManNAc kinase activity and sialylation in distal myopathy with rimmed vacuoles. J Biol Chem 2004;279:1 1402-7.

11 Eisenberg I, Grabov-Nardini G, Hochner H, et al. Mutations spectrum of GNE in hereditary inclusion body myopathy sparing the quadriceps. Hum Mutat 2003;21:99.

12 Hinderlich S, Berger M, Keppler OT, et al. Biosynthesis of N-acetylneuraminic acid in cells lacking UDP-N-acetylglucosamine 2-epimerase/ $\mathrm{N}$-acetylmannosamine kinase. Biol Chem 2001;382:291-7.

13 Neufeld MY, Sadeh M, Assa B, et al. Phenotypic heterogeneity in familial inclusion body myopathy. Muscle Nerve 1995;18:546-8. 